\title{
Pulmonary Function Test Reference Value
}

National Cancer Institute

\section{Source}

National Cancer Institute. Pulmonary Function Test Reference Value. NCI Thesaurus.

Code C128264.

A standardized value that allows for the comparison of an individual's pulmonary function test results to those same results obtained from a reference population, which establishes a context through which individual results can be interpreted. 